\title{
Hints of Cosmic Birefringence?
}

\author{
A new analysis of the cosmic microwave background shows that its \\ polarization may be rotated by exotic effects indicating \\ beyond-standard-model physics.
}

By Matteo Rini

$\mathrm{R}$

esearchers are increasingly scrutinizing the polarization of the cosmic microwave background (CMB)-the oldest

light in the Universe-for signs of new physics. One such sign would be a slight rotation of this polarization, as if the light had passed through a birefringent material with a polarization-dependent refractive index. This "cosmic birefringence" isn't predicted by the standard model and could arise from the coupling of CMB photons to hypothetical particles and fields. So far, experiments are consistent with zero birefringence, but their sensitivity has been hampered by certain systematic errors. Using an approach that mitigates such errors, Yuto Minami of the High Energy Accelerator Research Organization, Japan, and Eiichiro Komatsu of the Max Planck Institute for Astrophysics, Germany, and the University of Tokyo have analyzed CMB data from the Planck satellite and found tantalizing hints of a faint birefringence signal [1].

Systematic errors in tests of cosmic birefringence mostly derive from an unavoidable inaccuracy in the detector polarization angle. Since this inaccuracy $\alpha$ has the same effect as a

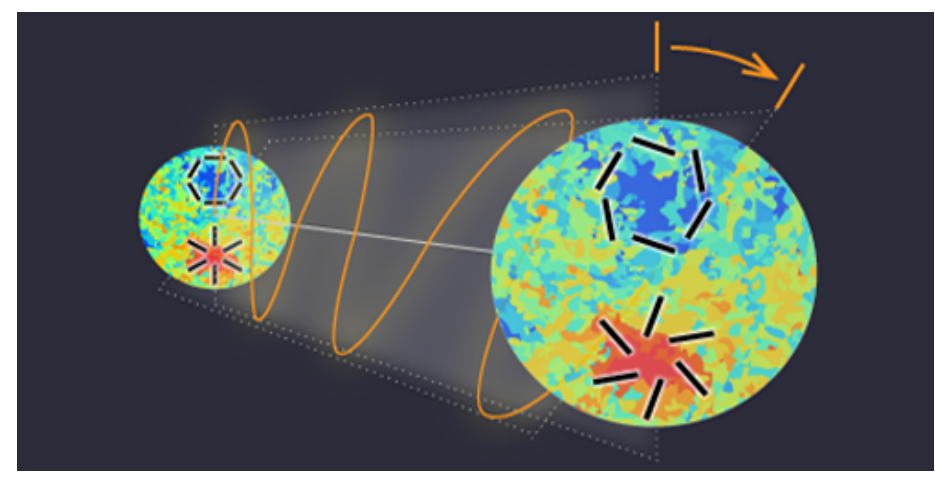

Credit: Y. Minami/KEK

cosmic-birefringence-induced rotation $\beta$, this miscalibration poses the biggest limit to detection sensitivity. Minami and Komatsu eliminated contributions from $\alpha$ by correlating CMB measurements (affected by both $\alpha$ and $\beta$ ) with measurements of foregrounds produced by microwave sources in our Galaxy (only affected by $\alpha$ ). Analyzing Planck data, they found a 99.2\% likelihood that $\beta$ is nonzero. This likelihood is a long stretch from the 5-sigma confidence needed for a discovery, but next-generation CMB detectors using the new method could strengthen the result. If confirmed, it would be a game-changing discovery, say the authors.

Matteo Rini is the Deputy Editor of Physics.

\section{REFERENCES}

1. Y. Minami and E. Komatsu, "New extraction of the cosmic birefringence from the Planck 2018 polarization data," Phys. Rev. Lett. 125, 221301 (2020). 